\title{
Colostrum Feeding Practices and Associated Factors Among Mothers Having Children Less Than 12 Months of Age in Wolaita Sodo City, Wolaita, Ethiopia 2019
}

\author{
Daniel Baza Gargamo \\ Department of Pediatrics and Neonatal Nursing, College of Health Science and Medicine, Wolaita Sodo University, Wolaita Sodo, Wolaita, \\ Ethiopia
}

\section{Email address:}

danielbaza9@gmail.com

\section{To cite this article:}

Daniel Baza Gargamo. Colostrum Feeding Practices and Associated Factors Among Mothers Having Children Less Than 12 Months of Age in Wolaita Sodo City, Wolaita, Ethiopia 2019. Biomedical Sciences. Vol. 6, No. 2, 2020, pp. 17-x24 doi: 10.11648/j.bs.20200602.11

Received: February 21, 2020; Accepted: March 4, 2020; Published: June 9, 2020

\begin{abstract}
Back ground: Colostrum is the principal milk produced in the first few days after delivery. It is a normative ordinary for the newborns concerning the comprehensive form of nutrition which is well thought -out as the "golden milk" is highly nutritious and contains anti-infective substances. It is very rich in vitamin A, protein and recommended as the newborns' absolute nourishment by UNICEF and WHO that should begin soon after delivery. The main purpose of this study was to assess colostrum feeding practices and associated factors among mothers having children less than 12 months age in Wolaita Sodo City, Wolaita, Ethiopia. Methods: A community-based cross-sectional study was employed from May 31 to Sep 312019 in Wolaita Sodo City. The data collected by using interview-based structured questionnaires among 396 eligible mothers through a systematic sampling technique. Data was analyzed by using SPSS version 25 . Both binary and multiple Variable logistic regressions ("odds ratio") analyses with a p-value $<0.05$ with 95\% confidence interval were identified as statistically significant. Result: Three hundred fifty seven, 87.4\% (95\%, CI: 11: 00- 14:00) of mothers practiced colostrum feeding after delivery. In multivariate logistic regression analysis at P-value of $<0.05$, Counseling on timely initiation of breastfeeding during antenatal care $(\mathrm{AOR}=7.798,95 \% \mathrm{CI}: 2.64-23.01)$, place of delivery $(\mathrm{AOR}=4.010,95 \% \mathrm{CI}: 1.260-12.760)$ and birth attendant of the current birth $(\mathrm{AOR}=3.119,95 \% \mathrm{CI}: 1.263-7.706)$ had the significant association with colostrum feeding practice. Conclusion: Counseling provided on early initiation of breastfeeding during antenatal care, giving birth at health care facilities and attending delivery by health care professionals were predictors of colostrum feeding among mothers having infants less than 12 months of age. Strengthening education on timely initiation of breastfeeding during antenatal care, promoting to give birth at health care institutions and accessing delivery attendance by health care workers are essential for colostrum feeding. Sustaining awareness creation strategies and approaches were suggested for the advancement of the nutritional value of colostrum and its health welfares for new born babies.
\end{abstract}

Keywords: Colostrum, Feeding Practice, Infants, Wolaita Sodo City, Wolaita, Ethiopia

\section{Introduction}

Child death is high internationally when equated to other age categories and greater than $75 \%$ happens in unindustrialized nations. Colostrum is the first liquid that is produced in the first few hours after delivery. Timely feeding of it to the newborn babies is continued as significant to challenge child nutrition and also the existence of newborn as revealed by scientific evidence [13]. Colostrum contain less amounts of lipid and potassium than usual milk [4, 5] It is helpful for bodily growth and intellectual development and has also laxative effect which encourages passage of baby's first stool which helps to prevent jaundice by clearing excess bilirubin that is created in large quantities at birth [1]. It contains various maternal immunoglobulins and is thick, sticky and clear to yellowish in color. It has proteins, vitamin A and maternal antibodies important to the newborn's nutrition until lactation is fully 
established and it has also high amounts of sodium, potassium, chloride, and cholesterol and various cytokine and growth factors which combination is supposed to encourage peak development of the infant's heart, brain, and central nervous system [1-4]. In a word it acts as a natural vaccine and ultimately reduces the leading causes of death like diarrhea and acute respiratory Infections (ARI) in developing nations of the World [1, 2]. Colostrum has a positive consequence in the prevention of juvenile malnutrition [1] and also delivers natural immunity (baby's first immunization) against many bacterial and viral infections through establishing important bacteria in the baby's gut [2-4]. Despite colostrum having these merits, it is rejected in some communities because it is taken as heavy, thick, dirty, toxic [1] and dangerous to children's health [1-4]. Inappropriately, colostrum feeding is not initiated to newborn soon after delivery for different communal mythologies and misunderstanding. These artificial problems distress directly and indirectly the health of newborn infants and results in malnutrition and high mortality rate in infants [1]. Suboptimal breastfeeding is accountable for $45 \%$ of newborn communicable mortality, $30 \%$ of diarrheal deaths and $18 \%$ of severe respiratory deaths among children less than five years of age $[1,2]$. In Ethiopia, about $18 \%$ of the infant deaths have been credited to deprived feeding practices [1-3]. Although colostrum feeding provides newborns with immunity to infection, any practice that reduces a frequency or volume of breastfeeding during this time could reduce an infant's long-term health and immunological defense $[1,2]$.

Even though the world health organization (WHO) recommended initiating colostrum feeding within the first hour of birth [1], a higher number of mothers avoided their colostrum before giving milk to their infant [1-5]. Mothers living in under developed countries avoid colostrum due to outdated views [1-3]. Prolonged labor, cesarean delivery, lack of knowledge regarding the benefits of colostrum and neonatal illness are the factors of colostrum's feeding [1-3]. Even though Ethiopia has developed the National Infant and Young Child Feeding guideline which recommends colostrum feeding $[1,2]$. There is wide variability towards colostrum feeding practice and discordant findings in different regions of Ethiopia [1-5] but no study was done on the feeding practices of colostrum and factors related to it in Wolaita Sodo City. Hence, this study aimed to investigate colostrum feeding practices and factors associated among mothers having children aged $<12$ months in Wolaita Sodo City, Wolaita, Ethiopia

\section{Methods}

\subsection{Study Area and Setting}

The study was carried out in Wolaita Sodo city. The city has a total population of 250521. From this, about 4752 mothers have infants less than 12 months of age. The city has three sub cities and 24 kebeles. Wolaita Sodo City has three health centers, one teaching and referral hospital owned by $\mathrm{MOH}$ and one private general hospital. The Wolaita Sodo city is located from $327 \mathrm{~km}$ from Addis Ababa, the capital of Ethiopia (11).

\subsubsection{Study Design and Period}

A community-based cross-sectional study was employed from May 31 to Sep 312019 in Wolaita Sodo City, Wolaita, Ethiopia

\subsubsection{Source Population}

All mothers having children less than 12 months of age in Wolaita Sodo city

\subsubsection{Study Population}

Selected mothers who have a child less than 12 months of age at the time of data collection in Wolaita Sodo City

\subsubsection{Inclusion Criteria}

Mothers who have a child less 12 months of age, resided in study area for at least six months and provided informed consent during the period of data collection

\subsubsection{Exclusion Criteria}

Mothers who were seriously ill and not volunteer to participate in the study

\subsection{Sample Size Determination}

Sample size was calculated with Epi info statistical software version 3.03 using single population proportion statistical formulas and considering colostrum feeding practice in Aksum town from the previous study as $86.5 \mathrm{n}=\mathrm{Z}$ $(1.96)^{2} \mathrm{P}(1-\mathrm{P}) / \mathrm{d}^{2,}$ where

$\mathrm{n}=$ the sample size $\int$

$\mathrm{z}=$ the level of confidence $(1.96)^{2}$ with $95 \% \mathrm{CI}$

$\mathrm{p}=$ the proportion of colostrum feeding practices of mothers having children less than 12 months age in Raya Kobo District $86.5 \%(12)$

$\mathrm{d}=$ margin of error

Sample size calculation:

Colostrum feeding practice $=86.5$

$\mathrm{n}=(z \propto / 2)^{2} . \mathrm{P}(1-\mathrm{P})=(1.96)^{2} .0 .865(1-0.865) \div(0.05)=180$

$\mathrm{d}^{2}$

Taking the design effect as 2 and considering $10 \%$ nonrespondent rate with confidence levels of $95 \%$ and $5 \%$ margin of error.

$\mathrm{nf}=$ final sample size was 396

\subsection{Sampling Procedure}

The multi stage sampling technique was used to select study population at the community level. First 8 kebeles were selected from the total of 24 kebeles. A sample size of 396 mothers who have a child with the age less than 12 months old from each kebele were selected based on proportional allocation to the sample size was done. Systematic sampling technique $(\mathrm{k}=12)$ was used to choose the household for an interview and the starting mother was selected using a lottery method. In the households with 
more than one child less than 12 months, the youngest child was selected randomly. The actual age of the infants was determined by asking the mothers and reviewing the birth date from vital certificate.

\subsection{Variable of Study}

\subsubsection{Dependent Variable}

Colostrum feeding practice.

\subsubsection{Independent (Exposure) Variables Related to} Maternal Socio-demographic and Economic Factors

Age of the mother, sex of the child, monthly income, family type, occupation of the mother, educational status of mother, educational status of father.

\subsubsection{Independent Variables Related to the Maternal Health Service Utilizations}

ANC follow up, counseling during ANC, birth order of the child, mode of delivery, place of delivery, birth attendants, and timely initiation of breastfeeding.

\subsubsection{Operational Definitions}

Colostrum: it is the yellowish breast milk created with in the first few days after delivery.

\subsection{Data Collection Instrument}

Structured, pre-tested and interviewer administered questionnaires were used. Questions on the questionnaire include the socio demographic characteristics, health service utilization, obstetrics and breast feeding related issues. The questionnaires were from the World Health Organization indicators for assessing infant and young child feeding practices and by adapting to the Ethiopian context.

\subsection{Data Collection Procedure}

Data were collected through face-to-face interview maintaining the predetermined sampling intervals by 5 professional nurses and 5 Health Officers. The principal investigator supervised data collectors. The data collectors informed the respondents all details of the research purpose and procedures and what was expected of them, potential risk and benefit in order to encourage accurate and honest response. When the mother was not available on the first visit, data collectors arranged alternative visits. If a woman was still not available on second visits or declined to participate in the study, the household was jumped and the immediate next household in the sampling frame was considered.

\subsection{Data Quality Management}

Before data collection, the questionnaire was first prepared in English and translated into Amharic and back to English to keep the consistency of questionnaire. Three days training was given to data collectors and supervisors by the principal investigator before the actual data collection.

A pretest was conducted in Bodit town on $5 \%$ of total sample size was tested. Based on the pretest, questionnaires were revised, edited and the necessary corrections made accordingly. The collected data were reviewed and checked for completeness and consistency by the supervisor and principal investigator on a daily basis.

\subsection{Data Processing and Analysis Procedures}

The data were coded, cleaned, entered and edited was done by using EPiData version 3.1 and exported to SPSS version 25 for analysis. Binary logistic regression analysis was employed to scan the statistical association between the outcome variable and every single independent variable. Variables which revealed statistical significance during bivariate analysis at $\leq 20 \%$ ( $p$-value $\leq 0.2$ ) were arrived into multivariate logistic regression to quarantine an independent effect of the predictors by using the backward removal method. The Hosmer-Lemeshow test was used to check the appropriateness of the model for analysis. Results were presented using tables and texts. Adjusted odds ratios (AOR) with $95 \% \mathrm{CI}$, were estimated to assess the strength of associations and statistical significance was stated at a pvalue $<0.05$

\subsection{Ethical Issues}

Ethical clearance was obtained from Wolaita Sodo University Institutional Review Board (IRB). Written consent was gotten from Wolaita Sodo city health department. During data collection all respondents were asked their authorization and informed consent was signed from each study participants.

\section{Results}

Socio-demographic and economic characteristics of the respondents

All eligible mothers of this study were agreed and participated in this study making the response rate of $(100.00 \%)$. The mean age of mothers participated in this study was 25.97 . A big majority $339(85.6 \%)$ of respondents were Wolaita in their ethnic group. About 275 (69.4) of respondents were protestant Christian in their religious affiliation. $151(38.1 \%)$ of mothers completed primary school and $298(75.3 \%)$ of them were housewives. Around 202 $(51 \%)$ of infants were males in their sex. From 396 mothers, $361(91.2 \%)$ of mothers were living with their nuclear family. The big majority of families 381 (96.21\%) families have less than three under-five children. About 350 (91.4\%) the study participants have exposure to mass media and majority of respondents $215(54.2 \%)$ of the families earn greater than or equal to 2500 hundred Ethiopian birr as monthly income (Table 1) 
Table 1. Soscio-demographic and economic characteristics of the respondents having infants of age less than 12 in Wolaita Sodo City, Wolaita, Ethiopia, 2019 $G C$.

\begin{tabular}{|c|c|c|}
\hline variables & Category $(n=396)$ & Frequency (\%) \\
\hline \multirow{4}{*}{ Age of the mother } & $<19$ & $27(6.8)$ \\
\hline & $20-24$ & $188(47.5)$ \\
\hline & $25-29$ & $111(28)$ \\
\hline & 35 and above & $24(6.1)$ \\
\hline \multirow{3}{*}{ Ethnicity } & Wolaita & $339(85.6)$ \\
\hline & Amhara & $23(5.8)$ \\
\hline & others & $13(3.3)$ \\
\hline \multirow{4}{*}{ Marital status of mother } & single & $2(.5)$ \\
\hline & Married & $369(93.2)$ \\
\hline & Divorced & $16(4)$ \\
\hline & Widowed & $9(2.3)$ \\
\hline \multirow{3}{*}{ Religious affiliation } & Protestant & $275(69.4)$ \\
\hline & Orthodox & $97(24.5)$ \\
\hline & Muslim & $22(5.6)$ \\
\hline \multirow{4}{*}{ Maternal educational level } & No formal education & $21(5.1)$ \\
\hline & Completed primary & $151(38.1)$ \\
\hline & Competed secondary & $138(34.8)$ \\
\hline & College and above & $87(22)$ \\
\hline \multirow{2}{*}{ Occupational status of mother } & House wife & $298(75.3)$ \\
\hline & employed & $98(24.7)$ \\
\hline \multirow{4}{*}{ Husbands educational status } & No formal education & $17(4.3)$ \\
\hline & Primary school & $98(24.7)$ \\
\hline & Secondary school & $133(33.6)$ \\
\hline & College and above & $123(31.1)$ \\
\hline \multirow{2}{*}{ Sex of infant } & Male & $202(51)$ \\
\hline & female & $194(49)$ \\
\hline \multirow{2}{*}{ Age of infant } & Birth to 6 months & $196(49.5)$ \\
\hline & 7 to 11 months & $200(50.5)$ \\
\hline Family type & Nuclear & $361(91.2)$ \\
\hline Number of under-five children & 4 and above & $15(3.78 \%)$ \\
\hline \multirow{2}{*}{ Exposure to mass media } & Exposed & $350(91.4)$ \\
\hline & Not exposed & $46(8.6)$ \\
\hline \multirow{5}{*}{ Monthly income } & Less than 1000 & $50(12.62)$ \\
\hline & 1000 to 1499 & $46(11.61)$ \\
\hline & 1500 to1999 & $35(8.83)$ \\
\hline & 2000 t0 2500 & $65(16.41)$ \\
\hline & Greater than or equal to 2500 & $215(54.29)$ \\
\hline
\end{tabular}

The respondents' obstetric and health care service utilization

The highest majority 379 (95.7) of respondents have received antenatal care. About 333 (86.9\%) of participants started their antenatal care before fifth month of gestation. Most of mothers 206 (54.3\%) have received antennal visit of four times. 249 (65\%) of the study participants have had got counseling on breast feeding. $226(57.1 \%)$ were receiving counseling on timely initiation of breastfeeding. $315(79.5 \%)$ respondents delivered at health institutions and 345 (87.1\%) of them assisted by health care professionals. From the total of 396 mothers, $347(87.6 \%)$ of mothers had spontaneous vaginal delivery. About $182(46 \%)$ of infants were first in their birth order (Table 2).

Table 2. Obstetric and health care service utilization among mothers' having infants less than 12 months of age in Wolaita Sodo City, Wolaita, Ethiopia 2019.

\begin{tabular}{|c|c|c|}
\hline variables & Categories or responses & Frequency $(\%)$ \\
\hline \multirow{2}{*}{ Antenatal visits } & Yes & $379(95.7)$ \\
\hline & No & $17(4.3)$ \\
\hline \multirow{2}{*}{ Gestational age at first antenatal visits $(n=379)$} & Before $5^{\text {th }}$ month & $333(86.9)$ \\
\hline & After $5^{\text {th }}$ month & $46(13.1)$ \\
\hline \multirow{3}{*}{ Number of antenatal visits (379) } & once & $21(5.8)$ \\
\hline & twice & $42(11.5)$ \\
\hline & four times & $206(54.3)$ \\
\hline
\end{tabular}




\begin{tabular}{|c|c|c|}
\hline variables & Categories or responses & Frequency (\%) \\
\hline \multirow{2}{*}{ Counseling on breast feeding during antenatal care $(\mathrm{n}=379)$} & Yes & $249(65)$ \\
\hline & No & $130(35)$ \\
\hline \multirow{2}{*}{ Counseling on timely initiation of breastfeeding during antenatal care $(n=379)$} & Yes & $226(57.1)$ \\
\hline & No & $173(42.9)$ \\
\hline \multirow{2}{*}{ Place of delivery $(n=396)$} & Health institution & $315(79.5)$ \\
\hline & Home & $81(20.5)$ \\
\hline \multirow{3}{*}{ Birth attendants $(\mathrm{n}=396)$} & Health care workers & $345(87.1)$ \\
\hline & Family & $32(8.1)$ \\
\hline & Traditional attendants & $19(4.8)$ \\
\hline \multirow{2}{*}{ Mode of delivery $(\mathrm{n}=396)$} & Spontaneous vaginal delivery & $347(87.6)$ \\
\hline & Caesarean section & $49(12.4)$ \\
\hline \multirow{3}{*}{ Infants birth order (396) } & First & $182(46)$ \\
\hline & Second & $121(30.6)$ \\
\hline & Third and above & $93(23.5)$ \\
\hline
\end{tabular}

Knowledge of mothers on colostrum feeding after delivery

From the total of 396 mothers involved in the study, 338 (83.1) heard about timely initiation of breastfeeding and 339 (85.6) thought that feeding breast milk is important for their infants. 300 (75.5) stated colostrum as the first vaccine for their children, 231 (58.3) reported that breastfeeding prevents uterine bleeding, $314(82.4)$ told that it can reduce neonatal mortality and 326 (78.3) declared that maternal breastfeeding increases the attachment between the mother and their baby (Table 3).

Table 3. Knowledge of mothers on colostrum feeding having children less than 12 months of age in Wolaita Sodo City, Wolaita, Ethiopia 2019 GC.

\begin{tabular}{lll}
\hline Variables (n=396) & responses & Frequency (\%) \\
\hline Colostrum is first vaccine & Yes & $300(75.75)$ \\
Think colostrum feeding & No & $96(24.5)$ \\
important) & Yes & $339(85.6)$ \\
Heard timely initiation of & No & $57(14.4)$ \\
breastfeeding & Yes & $338(83.1)$ \\
Early initiation of breast milk & No & $58(14.6)$ \\
prevents uterine bleeding & Yes & $231(58.3)$ \\
Timely initiation of breastfeeding & No & $165(41.7)$ \\
reduces neonatal mortality & No & $314(82.4)$ \\
Timely initiation of breast milk & Yes & $67(17.6)$ \\
increases attachment & No & $326(78.3)$ \\
\hline
\end{tabular}

Colostrum feeding practice and related characteristics of mothers

From the 396 mothers who participated in the study, 312 $(78.8 \%)$ initiated feeding their infants within one hour of delivery. About $346(87.4 \%)$ of mothers provided colostrum for their infants, $356(87.3 \%)$ were feeding their infants based on demand of the child (Table 4). months of age in Wolaita Soddo City, Wolaita, Ethiopia 2020 GC.

\begin{tabular}{lll}
\hline variables & responses & Frequency (\%) \\
\hline \multirow{2}{*}{ Fed colostrum for current child } & Yes & $346(87.4)$ \\
& No & $50(12.6)$ \\
Breast feeding current child & Yes & $382(96.5)$ \\
\multirow{2}{*}{ Breastfed with in 1 hour of delivery } & No & $14(3.5)$ \\
& Yes & $312(78.8)$ \\
Feeding based on demand of infant & Yes & $84(21.2)$ \\
& No & $347(87.6)$ \\
\hline
\end{tabular}

Factors associated with colostrum feeding practices

In the binary logistic regression at a $p$-value of $\leq 0.25$, family type, family income, exposure to mass media, ANC follow up, number of ANC follow up, counseling on timely initiation of breastfeeding during ANC visit, Early initiation of breastfeeding after delivery, place of delivery, birth order and birth attendant were statistically associated with colostrum feeding practice.

In multiple logistic regression by using backward elimination technique, mothers who have counseling on timely initiation of breastfeeding during antenatal care were 7.798 times higher to practice colostrum feeding than those mothers who have not counseled on timely initiation of breastfeeding $(\mathrm{AOR}=7.798,95 \% \mathrm{CI}$ : 2.64-23.01), mothers delivered at health care facilities were 4.010 times more to practice colostrum feeding than their counterparts. (AOR=4.010, 95\% CI: 1.260-12.760) and mothers attended by health care professionals have 3.119 times more likely to provide colostrum for their infants than those mothers assisted by non-health care workers $(\mathrm{AOR}=3.119,95 \% \mathrm{CI}$ : 1.263-7.706) had the significant association with colostrum feeding practice (Table 5).

Table 4. Colostrum feeding practice of mothers having children less than 12

Table 5. Factors associated with colostrum feeding practices among mothers having children less than 12 months of age in Wolaita Sodo City, Wolaita, Ethiopia, 2019 (Yes=Fed colostrum, No=Not fed colostrum).

\begin{tabular}{lccc}
\hline Colostrum feeding practice & & & \\
\hline Variables & Yes & No & COR (95\% CI) \\
\hline $\begin{array}{l}\text { Family type } \\
\text { Nuclear }\end{array}$ & 321 & 40 & $3(1.437-7.170) *$ \\
\hline
\end{tabular}




\begin{tabular}{|c|c|c|c|c|}
\hline \multicolumn{5}{|l|}{ Colostrum feeding practice } \\
\hline Variables & Yes & No & COR $(95 \%$ CI) & AOR (95\% CI) \\
\hline Extended & 25 & 10 & 1 & 1 \\
\hline \multicolumn{5}{|l|}{ ANC follow up } \\
\hline Yes & 327 & 42 & $7(2.611-19.483) *$ & $0.00(0.001-0.056)$ \\
\hline No & 9 & 8 & 1 & 1 \\
\hline \multicolumn{5}{|l|}{ Exposure to mass media } \\
\hline Yes & 321 & 41 & $2.819(1.231-6.453)^{*}$ & $0.254(0.052-1.250)$ \\
\hline No & 25 & 9 & 1 & 1 \\
\hline \multicolumn{5}{|l|}{ Counseled on EXBF } \\
\hline Yes & 230 & 17 & $9.840(3.494-27.711)^{*}$ & $0.529(0.102-2.741)$ \\
\hline No & 11 & 8 & 1 & 1 \\
\hline \multicolumn{5}{|l|}{ Counseled on TIBF } \\
\hline Yes & 212 & 14 & $5.744(2.383-13.846)^{*}$ & $7.798(2.64-23.01) * *$ \\
\hline No & 29 & 11 & 1 & 1 \\
\hline \multicolumn{5}{|l|}{ Breastfed with in 1 hour } \\
\hline \multicolumn{5}{|l|}{ Of delivery } \\
\hline Yes & 288 & 24 & $5.37(2.87-10.024)$ & $0.039(0.086-0.066)$ \\
\hline No & 58 & 26 & 1 & 1 \\
\hline \multicolumn{5}{|l|}{ Place of birth } \\
\hline Health institution & 291 & 24 & $0.174(0.093-0.326)^{*}$ & $4.010(1.260-12.760)^{* *}$ \\
\hline Home & 55 & 26 & 1 & 1 \\
\hline \multicolumn{5}{|l|}{ Birth attendant } \\
\hline Health care professional & 318 & 27 & $3.472(2.353-5.122$ & $3.119(1.263-7.706)^{* *}$ \\
\hline Non health care professional & 28 & 23 & 1 & 1 \\
\hline
\end{tabular}

NB: $*$ P-value $=0.2, * * \mathrm{P}$-value $=0.001$.

\section{Discussion}

The main reason of this particular study was to assess colostrum feeding practice and its associated factors amongst mothers having infants less than 12 months age in Wolaita Sodo City. This study has shown that the magnitude of colostrum feeding practice was $87.4 \%$ which is consistent with the finding reported from Raya Kobo district, Kombolcha town and North Wollo Zone respectively [20, 25, 27]. The result of this study is lower than similar study done at Aksum town which was found to be $94.7 \%$ [26]. The finding from the current study is almost similar to the report from North Wollo zone which was $88 \%$ [25]. The finding of recent study was greater than similar studies done at Debre Tabor town [28] and Northern part of Ethiopia [13]. The differences among these current and previous studies may be due to period of the study, socio-demographic and economic disparities, socio-cultural variability among the study participants, differences in the study design and sample size.

The finding from current study is higher than the similar studies done in different developing countries which was $79 \%$ in Assam, India [19], 63\% in Bangladesh [29], 69\% in Nepal and $83 \%$ in South Africa $[29,30]$. The result from the recent study is lower than similar study in Burkina Faso which showed colostrum feeding $92 \%$ and $97 \%$ in Uganda respectively [30]. The differences may be due to crosscultural, educational, infant feeding styles and residential dissimilarities.

Counseling given for prenatal mothers on timely initiation of breastfeeding during antenatal follow up heightens the feeding practice of colostrum. The mothers who got counseling on early initiation of breastfeeding practiced colostrum feeding 7.798 times higher than their counterparts. The finding is similar to studies in the Northern part of Ethiopia [25, 26]. This may be due to creation of awareness towards advantage of colostrum to their infants during promotion of timely breastfeeding initiation. Recommending timely breastfeeding during pregnancy may also keep the place for mothers to discuss the essentials of colostrum for their newborn critically.

The odds of colostrum feeding practice among mothers who delivered at health care facilities were 4.010 times higher than those who gave their current birth at home. This is in line with the study done at North Wollo Zone [25]. The lower odds of colostrum feeding at home delivery could be due to the pressure from family members, friends or significant others. The higher odds of mothers who delivered at health institutions can be because of information from the birth attendants, counseling on colostrum advantages during immediate postnatal period or delayed breastfeeding initiation after delivery.

\section{Conclusion}

Counseling provided on early initiation of breastfeeding during antenatal care for antenatal care mothers, place of delivery and birth attendants were predictors of colostrum feeding practice among mothers having infants less than 12 months of age. Strengthening education on timely initiation of breastfeeding during antenatal care, promoting to give birth at health care institutions and accessing delivery attendance by 
health care workers are essential for colostrum feeding. Sustaining awareness creation strategies and approaches were suggested for the advancement of the nutritional value of colostrum and its health welfare for new born babies.

Based on the finding from current study, it is better to provide culturally tailored health education for pregnant mothers by health extension workers focusing on the essentials of colostrum feeding for the betterment of their babies.

\section{Strength of the Study}

The study tried to address mothers recall bias by limiting the infants age to less than 12 months. Rather than facility based study, community centered study was used to reduce the social desirability bias. The protective relationship between colostrum feeding practices and risk was demonstrated after controlling for factors that are known to be associated with colostrum feeding experiences. This particular study also tried to limit sample related issues. Another strength may be, this is the first study on colostrum feeding at the study area in particular to address issues related early breastfeeding problem

\section{Limitations}

The evidence obtained from mothers might be exposed to memory bias since the data were gathered grounded on selfreport of the mothers of infants aged less than 12 months age. Lack of support with qualitative data may be another short back. The study also shares some constraints of crosssectional study design.

\section{Abbreviations}

UNICEF: united Nations children's fund

WHO: world health organization

SPSS: statistical software for social sciences

P-value: probability value

CI: confidence interval

AOR: adjusted odds ratio

ARI: acute respiratory infections

IYCF: infants and young child feeding

$\mathrm{MOH}$ : minister of health

ANC: antenatal care

Epi info: epidemiological information

IRB: institutional review board

\section{Declarations}

\section{Ethical Approval and Consent to Participate}

The ethical clearance for this study was proven by the Wolaita Sodo University institutional review board and a supportive letter was obtained from the Wolaita Sodo City health department office. The study participants have fully informed on the purpose of the study and information was collected after obtaining written informed consent from each participant. Information was documented secretly and confidentiality was guaranteed during the study period.

\section{Data availability and Materials}

The datasets used during the current study are available from the corresponding author on reasonable request.

\section{Conflicts of Interest}

The author declare that there is no conflict of interest.

\section{Funding Source}

This study has no funding source.

\section{Acknowledgements}

I would like thank Wolaita Sodo University College of Health Science and Medicine, Wolaita Sodo City Health Department, the supervisors, data collectors and the study participants for their valuable support, facilitation and fully involvement on the progress of this study.

\section{References}

[1] Abt Associates Inc. CLEB, Philip J. Smith, and Larry Wilkinson. National Immunization Survey August 2005; Guideline.

[2] P. Early interruption of exclusive breastfeeding: results from the eight-country MAL-ED study. Journal of Health, Population and Nutrition. 2015; 34 (10).

[3] al SAe. Exclusive Breastfeeding Reduces Acute Respiratory Infection and Diarrhea Deaths Among Infants in Dhaka Slums. American Academy of Pediatrics. 2001; 108 (5).

[4] al. He. Breastfeeding in infancy: identifying the programrelevant issues in Bangladesh. International Breastfeeding Journal. 2010; 5 (1).

[5] al. T. Suboptimal breastfeeding of infants during the first six months and associated factors in rural communities of Jimma Arjo Woreda, SouthwestEthiopia. BMC Public Health. 2012; $363(363)$

[6] Carneiro-Sampaio ILGFJA-BGDLTM. Human colostrum contains IgA antibodies reactive to enteropathogenic Escherichia coli virulence-associated proteins: intimin, BfpA, EspA, and EspB. Journal of Pediatric Gastroenterology and Nutrition. AUGUST 1998; 27, (2): 166-71.

[7] JE Lawn KK, C Enweronu-Laryea, O Massee Bateman. Newborn survival in low resource settings - are we delivering? BJOG An International Journal of Obstetrics and Gynaecology. 2009; x: 49-59.

[8] UNICEF. A fair chance for every child: 70 years for every child. Guideline. 2016

[9] World Health Organization UaWI. BABY-FRIENDLY HOSPITAL INITIATIVERevised, Updated and Expanded for Integrated Care. 2009. 
[10] Dat V Duong CWBAHL. Breast-feeding initiation and exclusive breast-feeding in ruralVietnam. Public Health Nutrition. 2004; 7 (6): 795-99.

[11] Gribble KD. Mental health, attachment and breastfeeding: implications for adopted children and their mothers. International Breastfeeding Journal. 2006; 1 (5).

[12] Joshi DSK. Colostrum Feeding: Knowledge, Attitude andPractice in Pregnant Women in a Teaching Hospital in Nepal.

[13] NL Rogers et al.: Colostrum avoidance, prelacteal feeding and late breast-feeding initiation in northern Ethiopia. Journal of public health nutrition, 14 (11): 2011, p. 2029-2036.

[14] Expansion of the Baby-Friendly Hospital Initiative Ten Steps to Successful Breastfeeding into Neonatal Intensive Care: Expert Group Recommendations Journal of human lactation. May 31, 2013; 29 (3).

[15] Kliegman. RM. Nelson Textbook of Paediatrics. 20 th ed. 2016.

[16] Organization WH. Mortality and burden of disease attributable to selected major risks. 2009.

[17] Unicef. Committing to Child Survival: A GLOBAL MOVEMENT TO END PREVENTABLE CHILD DEATHS. Progress Report 2014; Progress Report.

[18] EDMOND ea. Delayed Breastfeeding Initiation Increases the Risk of Neonatal Mortality. journal of the American Academy of Pediatrics.

[19] al KRe. Colostrum feeding practices and its determinants among urban and rural mothers in Kamrup, Assam, India. International Journal of Research in Medical Sciences Int J Res Med Sci Oct; 4 (10): 4567-4572 www msjonline org. 2016; 4 (10): 4567-72.

[20] Misgan Legesse MD, Firehiwot Mesfin and Demewoz Haile. Factors Associated with Colostrum Avoidance Among Mothers of Children Aged less than 24 Months in Raya Kobo district, North-eastern Ethiopia: Community-based Crosssectional Study. Journal of Tropical Pediatrics. 2015.

[21] al Ne. Expansion of the Baby-Friendly Hospital Initiative Ten Steps to Successful Breastfeeding into Neonatal Intensive
Care: Expert Group Recommendations. Journal of Human Lactation. 2018; 29 (3): 300-9.

[22] February 27. Breastfeeding and the Use of Human Milk. February 27, 2012. February 27, 2012.

[23] Disha AD R, Subandoro and $\mathrm{P}$ Menon. INFANT AND YOUNG CHILD FEEDING (IYCF) PRACTICES IN ETHIOPIA AND ZAMBIA AND THEIR ASSOCIATION WITH CHILD NUTRITION: ANALYSIS OF DEMOGRAPHIC AND HEALTH SURVEY DATA. African Journal of food, nutrition, agriculture and development. 2012; $12(2)$.

[24] Liben Ya, 10. D. Effects of home delivery on colostrum avoidance practices in North Wollo zone, an urban setting, Ethiopia: a cross sectional study. Journal of Health, Population and Nutrition. 2018; 37 (4).

[25] We, https://doi.org/10.1186/s13104-018-3712-z. Colostrum avoidance and associated factors among mothers having children less than 2 years of age in Aksum town, Tigray, Ethiopia: a cross-sectional study 2017. BMC Research Notes. 2017; 11 (601).

[26] COLOSTRUM AVOIDANCE AND ASSOCIATED FACTORS AMONG MOTHERS OF CHILDREN AGED LESS THAN 12 MONTHS IN KOMBOLCHA TOWN, SOUTH WOLLO ZONE, ETHIOPIA.

[27] Goshu Aa. Early initiation of breastfeeding and colostrum feeding among mothers of children aged less than 24 months in Debre Tabor, northwest Ethiopia: a cross-sectional study. BMC Research Notes. 2019; 12 (65).

[28] Islam MS KR. Colostrum feeding status in a selected rural area of Bangladesh. Journal of Nutritional Health \& Food Engineering. 2019; 9 (2): 52-4.

[29] Joshi S BB, Lamsal S. Colostrum Feeding: Knowledge, Attitude andPractice in Pregnant Women in a Teaching Hospitalin Nepal. Web Central. 2012.

[30] http://www.internationalbreastfeedingjournal.com/content/9/1/ $19 \mathrm{Ea}$. Early infant feeding practices in three Africancountries: the PROMISE-EBF trial promotingexclusive breastfeeding by peer counsellors. International Breastfeeding Journal. 2014; 9. 